\title{
RNA granules: the clock within
}

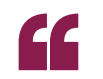

translation

timing in

vertebrate

oocytes

depends

on the

assembly and

disassembly of

RNA granules

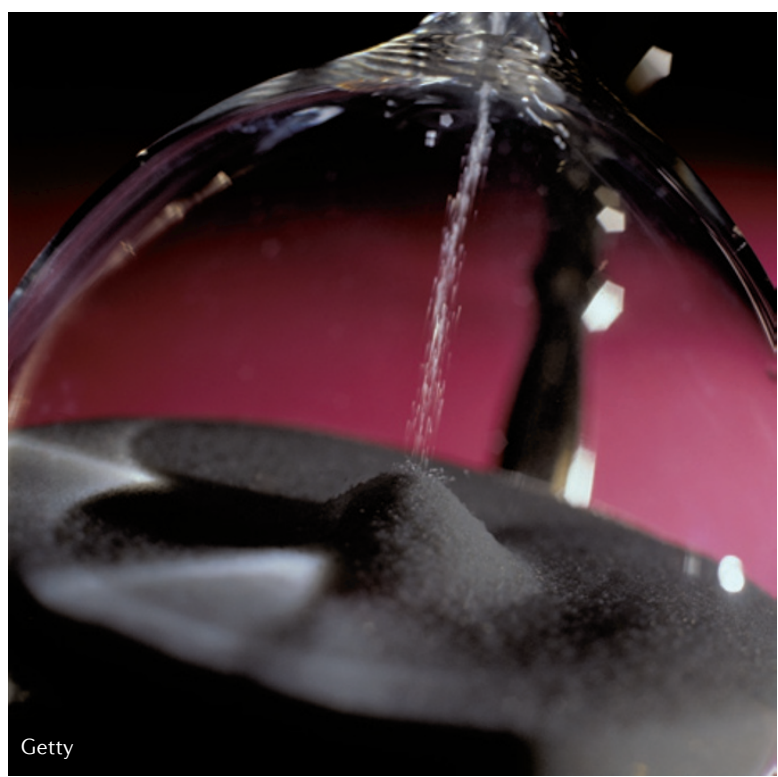

accumulate in the cytoplasm. Upon receiving specific signals (including hormones) that induce oocyte maturation, these stored mRNAs are translated in a specific order that mediates progression to meiotic metaphase II, preparing oocytes for fertilization. Temporally controlled translation of cyclin B mRNAs is key to oocyte maturation, as it induces germinal vesicle breakdown (GVBD), assembly of the first meiotic spindle and polar body extrusion.

The authors first observed that cyclin B1 mRNA is deposited in cytoplasmic granules that are asymmetrically distributed in mouse and zebrafish oocytes. After induction of oocyte maturation, cyclin B1 mRNA levels remained constant, but the number of granules decreased from prophase I to prometaphase I and almost disappeared by the time oocytes reached metaphase II. Importantly, this granule disassembly coincided with the activation of cyclin B1 mRNA translation.

Kotani et al. then analysed how these RNA granules form and whether they might affect translational control. They found that the Pumilio 1 (PUM1) protein (known to regulate cyclin $\mathrm{B} 1$ translation in frog oocytes) bound to cyclin B1 mRNAs in both zebrafish and mouse oocytes and that this binding was necessary for the formation of cyclin B1 mRNA granules. Furthermore, although PUM1 was not required for translational repression, its binding regulated the timing of cyclin B1 mRNA translational activation; mutant mRNAs unable to bind PUM1 were translated earlier than wild-type mRNAs following induction of oocyte maturation. Treatment with cytochalasin D, which promotes F-actin depolymerization, revealed that granule formation also depends on F-actin. Moreover, F-actin stabilization prevented granule disassembly, cyclin B1 synthesis and GVBD.

This work shows that cyclin B1 RNA granules, the formation of which depends on PUM1 and F-actin, keep cyclin B1 mRNA in a repressed state until the appropriate time during oocyte maturation and provides evidence that translation timing in vertebrate oocytes depends on the assembly and disassembly of RNA granules. The molecular mechanisms underlying granule-mediated translational control remain to be elucidated.

Kim Baumann

ORIGINAL RESEARCH PAPER Kotani, T.et al.

Cyclin B1 mRNA translation is temporally

controlled through formation and disassembly of RNA granules.J. Cell Biol. http://dx.doi. org/10.1083/jcb.201302139 (2013) 